\title{
Constant Congestion Routing of Symmetric Demands in Planar Directed Graphs
}

\author{
Chandra Chekuri*1, Alina Ene ${ }^{2}$, and Marcin Pilipczuk ${ }^{\dagger 3}$ \\ 1 Department of Computer Science, University of Illinois, Urbana-Champaign, \\ USA \\ chekuri@illinois.edu \\ 2 Department of Computer Science and DIMAP, University of Warwick, \\ Warwick, UK \\ A.Ene@dcs.warwick.ac.uk \\ 3 Institute of Informatics, University of Warsaw, Warsaw, Poland \\ malcin@mimuw.edu.pl
}

\begin{abstract}
We study the problem of routing symmetric demand pairs in planar digraphs. The input consists of a directed planar graph $G=(V, E)$ and a collection of $k$ source-destination pairs $\mathcal{M}=$ $\left\{s_{1} t_{1}, \ldots, s_{k} t_{k}\right\}$. The goal is to maximize the number of pairs that are routed along disjoint paths. A pair $s_{i} t_{i}$ is routed in the symmetric setting if there is a directed path connecting $s_{i}$ to $t_{i}$ and a directed path connecting $t_{i}$ to $s_{i}$. In this paper we obtain a randomized poly-logarithmic approximation with constant congestion for this problem in planar digraphs. The main technical contribution is to show that a planar digraph with directed treewidth $h$ contains a constant congestion crossbar of size $\Omega(h / \operatorname{polylog}(h))$.
\end{abstract}

1998 ACM Subject Classification F.2.2 Nonnumerical Algorithms and Problems, G.2.2 Graph Theory

Keywords and phrases Disjoint paths, symmetric demands, planar directed graph

Digital Object Identifier 10.4230/LIPIcs.ICALP.2016.7

\section{Introduction}

Disjoint path problems are well-studied routing problems with several applications and fundamental connections to algorithmic and structural results in combinatorial optimization and graph theory. Canonical problems here are the edge-disjoint paths problem (EDP) and the node-disjoint paths problem (NDP) in undirected graphs. In both these problems the input consists of an undirected graph $G=(V, E)$ and $k$ node-pairs $\left\{s_{1} t_{1}, \ldots, s_{k} t_{k}\right\}$. In EDP the goal is to connect the pairs by edge-disjoint paths and in NDP the goal is to connect the pairs by node-disjoint paths. The decision versions of these problems are NP-Complete when $k$ is part of the input. The seminal work of Robertson and Seymour showed that both these problems are fixed parameter tractable when parameterized by $k$, the number of pairs. In this paper we are concerned with an optimization version of the problems where the goal is to maximize the number of input pairs that can be routed via edge or node-disjoint

\footnotetext{
* Supported in part by NSF grant CCF-1319376.

$\dagger$ Research done while the author was at University of Warwick, partially supported by DIMAP and by Warwick-QMUL Alliance in Advances in Discrete Mathematics and its Applications.
} 
paths. To avoid notational overload we will henceforth use EDP and NDP to refer to these maximization versions.

The approximability of EDP and NDP has been extensively studied but our understanding is still limited. The best known approximation for both these problems is $O(\sqrt{n})[9,31]$ (here $n$ is number of nodes in $G$ ) while current hardness of approximation results only rule out an $O\left(\log ^{1 / 2-\varepsilon} n\right)$ approximation [2]. Even in planar graphs the best approximation up to very recently was $O(\sqrt{n})$, with a slight improvement just announced [16]. One of the reasons for this state of affairs is that the natural multicommodity flow relaxation has an integrality gap of $\Theta(\sqrt{n})$. On the other hand, two closely related relaxations of these problems have seen significant progress in the last decade. ANF is the relaxation of the disjoint paths problem where a subset of the input pairs $\mathcal{M}^{\prime}$ is routed if there is a feasible multicommodity flow in the graph that routes one unit of flow for each pair in $\mathcal{M}^{\prime}$. A second relaxation is to allow some small constant congestion $c$, i.e., instead of the pairs being routed on disjoint paths we allow up to $c$ paths to use a given edge or node. ANF admits a poly-logarithmic approximation $[11,8]$. A series of breakthroughs [33, 1, 13] culminated in a poly-logarithmic approximation for EDP with congestion 2 by Chuzhoy and Li [17]. These ideas have been extended to NDP as well $[6,4]$. These results have been made possible by a number of non-trivial ideas and techniques at the intersection of algorithms, combinatorial optimization and graph theory. In particular, the results have been enabled by and contributed to a deeper understanding of the structure of undirected graphs via the notion of treewidth. Treewidth is a well-known graph parameter that plays a fundamental role in the graph-minor theory of Robertson and Seymour; see $[3,4,5,14]$ for some of the recent results.

It is natural to study disjoint paths problems also in directed graphs. Here the graph $G$ is directed and the input pairs $\mathcal{M}=\left\{\left(s_{1}, t_{1}\right), \ldots,\left(s_{k}, t_{k}\right)\right\}$ are ordered and we seek to find a maximum cardinality subset of $\mathcal{M}$ that can be connected by disjoint paths ${ }^{1}$. Unfortunately, it has been shown that disjoint paths problems are highly intractable in directed graphs. It is known that even the simpler case of ANF and with congestion $c$ allowed is hard to approximate to within a factor of $n^{\Omega(1 / c)}$ [15]; moreover this holds in acyclic graphs.

A recent paper by a subset of the authors [7] initiated the study of maximum throughput routing problems in directed graphs where the demand pairs are symmetric. Here the graph $G$ is directed but the input pairs are unordered as in the undirected setting. Routing a pair $s_{i} t_{i}$ requires finding a path that connects $s_{i}$ to $t_{i}$ and a path connecting $t_{i}$ to $s_{i}$. We use Sym-Dir-EDP, Sym-Dir-NDP and Sym-Dir-ANF to denote the analogues of EDP, NDP and ANF respectively in this setting. A detailed motivation for the study of this model is given in [7]. Here we briefly outline some of the key points.

The model is motivated by both theoretical and practical considerations. On the theoretical side the model generalizes (modulo constant congestion) the edge and node disjoint paths problems in undirected graphs. Moreover, flow-cut gaps in this model have been studied in the past and have close connections to various problems including feedback edge/vertex set problems $[30,37,21,10]$. From the more practical side there are several scenarios where the communication between users is symmetric while the underlying network that supports the communication may be asymmetric (hence modeled as a directed graph); see [26, 25] for instance.

Unlike the case of directed graph routing problems, the symmetric model exhibits tractability. In particular, the well-linked decomposition framework for undirected graphs extends to a large extent to this model [7].

1 Although edge and node disjoint paths problems are equivalent in general directed graphs, this is not necessarily the case in restricted graph classes such as planar graphs. 
To resolve the complexity of disjoint path problems in the symmetric model one needs to understand the structure of directed graphs as a function of their directed treewidth [23, 34], that we denote by $\operatorname{dtw}(G)$. As we mentioned, the interplay between algorithmic questions and graph structure theory for undirected graphs has been very successful in the recent past. There has been recent significant progress on the graph theoretic side on directed treewidth; in particular Kawarabayashi and Kreutzer recently established the excluded grid theorem in directed graphs [27, 28].

The main technical contribution of [7] is to generalize the well-linked decomposition framework of [8] to the symmetric demands setting in directed graphs. As a consequence, [7] obtained a poly-logarithmic approximation with constant congestion for Sym-Dir-ANF. The central open question they raised is the following: Is there a poly-logarithmic approximation for Sym-Dir-NDP with constant congestion in general directed graphs? It was shown in [7] that this can be answered in the positive by addressing the following question which is the analogue that was raised in [8] for undirected graphs: If a directed graph $G$ has directed treewidth $h$, does it have a constant congestion routing structure (crossbar) of size $\Omega(h / \operatorname{polylog}(h))$ ? Note that grid-minor theorems establish such a connection between treewidth and routing structures, however, the quantitative relationship between the treewidth and the size of the grid is too weak to prove any meaningful approximation for the routing problem. On the other hand, the routing problem has the flexibility of allowing a large constant congestion which enables one to prove the existence of routing structures that are not as rigid as a grid; this relaxation has been the key to algorithmic success on routing. We also note that it is NP-Complete to decide whether a single pair can be routed without congestion in the symmetric setting [22]; thus a congestion of at least 2 is necessary for a non-trivial approximation ratio.

In this paper we take a step towards the general problem by addressing the important special case of planar graphs. Our main algorithmic result is the following.

- Theorem 1. There is a randomized poly-logarithmic approximation for Sym-Dir-NDP in planar directed graphs with congestion 5.

The approximation algorithm in the preceding theorem is derived via a natural multicommodity flow relaxation for the problem. The main new technical ingredient is a graph theoretic result that shows that if a planar digraph has directed treewidth $h$ then it has a constant congestion crossbar of size $\Omega(h /$ polylog $(h))$. We remark that an undirected planar graph with treewidth $h$ has a grid-minor (which is a congestion 2 crossbar) of size $\Omega(h)$. In contrast the known relationship between treewidth and grid-minors in directed planar graphs is much weaker; recent work $[27,28]$ only shows that there is a directed-grid of size $f(h)$ for some weakly growing function of $h$. We hope that our crossbar result could be used as a starting point to improve the quantitative bound on the grid-minor theorem for planar digraphs.

\subsection{Overview of the Algorithm and Technical Contributions}

Here we give a brief outline of the high-level details of the algorithm and some of our technical contributions. Let $(G, \mathcal{M})$ be an instance of Sym-Dir-NDP, where $G=(V, E)$ is a directed planar graph with unit node capacities, and $\mathcal{M}=\left\{s_{1} t_{1}, \ldots, s_{k} t_{k}\right\}$ is a collection of source-destination pairs. We refer to the nodes participating in $\mathcal{M}$ as terminals, and we use $\mathcal{T}$ to denote the set of terminals. It is convenient to assume that the pairs $\mathcal{M}$ form a matching on $\mathcal{T}$. 
Well-linked sets: A key notion that we make use of is well-linkedness. Given a directed graph $G=(V, E)$ a subset of nodes $X \subseteq V$ is said to be well-linked if for any two disjoint subsets $Y$ and $Z$ of $X$ of equal size, there exist $|Y|$ node-disjoint paths from $Y$ to $Z$; note that the definition is symmetric since we can swap $Y$ and $Z$. We need a relaxation of well-linkedness. For some parameter $\beta \in[0,1], X$ is $\beta$-well-linked if for all disjoint $Y, Z \subset X$ of equal size there are $|Y|$ paths from $Y$ to $Z$ such that no node is in more than $\lceil 1 / \beta\rceil$ of these paths; in other words, the node-congestion caused by the paths is at most $\lceil 1 / \beta\rceil$. The case $\beta=1$ corresponds to well-linkedness. It is well-known that in both directed and undirected graphs well-linkedness is closely connected to treewidth. More precisely, a graph has treewidth $k$ iff it has a well-linked set of size $\Theta(k)$; see [34]. Moreover, if $X$ is $\beta$-well-linked in $G$ then the treewidth of $G$ is $\Omega(\beta|X|)$.

Algorithm: Here we outline the high-level steps of our algorithm.

1. Solve a multicommodity flow based LP relaxation that routes each pair $s_{i} t_{i}$ fractionally to an amount $x_{i} \in[0,1]$ to maximize $\sum_{i=1}^{k} x_{i}$. See Fig. 2 and the description in Section 2.

2. Use the LP relaxation and the well-linked decomposition framework from [7] to reduce the problem, at the loss of a poly-logarithmic factor in the approximation, to instances in which the terminals $\mathcal{T}$ are $\alpha$-well-linked for some fixed constant $\alpha$.

3. Assuming that $\mathcal{T}$ is $\alpha$-well-linked in $G$ we have $\operatorname{dtw}(G)=\Omega(k)$ where $k=|\mathcal{T}|$. Using this fact show that $G$ has a large routing structure and use this structure to route a large number of terminal pairs. Use the following steps.

a. From $G$ obtain an Eulerian multigraph $H=\left(V, E_{H}\right)$ whose support is a subgraph of $G$ such that (i) $\mathcal{T}$ is $\alpha^{\prime}$-well-linked in $H$ for $\alpha^{\prime}=\Omega\left(\frac{1}{\operatorname{polylog}(k)}\right)$ and (ii) $\Delta(H)$, the maximum degree in $H$, is $\operatorname{polylog}(k)$.

b. Using the fact that $H$ is Eulerian, has treewidth $\Omega(k / \operatorname{polylog}(k))$, and has maximum degree polylog $(k)$, show that it has a cylinder-like routing structure of size $\Omega(k / \operatorname{polylog}(k))$. See Fig. 1 .

c. Route terminals to the routing structure and use it to connect a large number of input pairs.

The preceding algorithm follows the general framework that has been very successful in the undirected graph setting in the recent past. The first two steps follows the well-linked decomposition framework from [8] that has been extended to the symmetric demand instances in directed graphs by [7]. This framework allows one to reduce, via the LP relaxation, general instances to instances in which the terminals are well-linked. This incurs a poly-logarithmic factor loss in the approximation. With this reduction in place we have the following property for our instance. The graph $G$ has a terminal set $\mathcal{T}$ of size $h$ and since $\mathcal{T}$ is $\alpha$-well-linked for some fixed constant $\alpha, G$ has directed treewidth $\Omega(h)$. Now, the remaining task is to show a graph-theoretic result that any directed graph with treewidth $h$ has a constant congestion crossbar routing structure of size $\Omega(h / \operatorname{polylog}(h))$. By crossbar we mean a directed graph $H$ with an interface $I \subset V(H)$ with the following property: any matching on $I$ can be routed in a symmetric fashion in $H$ with constant congestion. The idea then is to route the terminals to the interface of the crossbar and use it to route the desired matching on the terminals.

In undirected planar graphs if $G$ has treewidth $h$ then it has grid-minor of size $\Omega(h)$ [36], and this grid-minor can be used as a crossbar to route $\Omega(h)$ input pairs (see [8] for instance). What about directed graphs? Johnson et al. [23], who introduced the notion of directed treewith, conjectured that any directed graph with sufficiently large treewidth contains a cylindrical grid (see Fig. 1) as a butterfly minor. The cylindrical grid can be used as a 
crossbar. In an unpublished manuscript, Johnson et al. [24] outlined a proof for the case of planar graphs. Kawarabayashi and Kreutzer [27] recently gave a different proof for the planar and minor-free case, and very recently gave a proof for all graphs [28]. However, as we already mentioned, the quantitative relationship between the size of the cylindrical grid and treewidth is very weak. Hence, these results would not yield any meaningful results for our routing problem. Here, we build on the high-level ideas in the work of Johnson et al. [24] to establish our main result which gives a constant congestion crossbar of size $\Omega(h / \operatorname{polylog}(h))$ where $h$ is the treewidth of $G$; our result applies only to planar graphs and establishing a similar result for general graphs is a challenging open problem. Due to space constraints we only mention the key steps.

A key insight from [24] is that given directed graph $G$ one can create an Eulerian multigraph $H$ of bounded degree whose support is a subgraph of $G$ such that $\operatorname{dtw}(H) \geq$ $f(\operatorname{dtw}(G))$ for some function $f$. Eulerianness as well as small degree are critical for further manipulations. Our first contribution is to show that $H$ can be chosen such that (i) dtw $(H)=$ $\operatorname{dtw}(G) / \operatorname{polylog}(\operatorname{dtw}(G))$ and (ii) the maximum degree in $H, \Delta(H)=O\left(\log ^{2} \operatorname{dtw}(G)\right)$. For this purpose we use Louis's extension of the cut-matching game of Khandekar, Rao and Vazirani [29] to directed graphs [32], combined with the well-linked decomposition framework of $[7,8]$.

- Theorem 2. Suppose that there is a polynomial time algorithm for $\Omega(1)$-node-well-linked instances of Sym-Dir-NDP in planar directed Eulerian graphs of maximum degree $\Delta$ that achieves a $\beta(\Delta)$-approximation with congestion $c$. Then there is a polynomial time randomized algorithm that, with high probability, achieves a $\beta\left(O\left(\log ^{2} k\right)\right) \cdot O\left(\log ^{6} k\right)$ approximation with congestion $c$ for arbitrary instances of Sym-Dir-NDP in planar directed graphs, where $k$ is the number of pairs in the instance.

Another key insight from [24] is to consider the undirected version of $G$, denoted by $G^{\mathrm{UN}}$, to obtain a large undirected grid-minor using the fact that $\operatorname{tw}\left(G^{\mathrm{UN}}\right)=\Omega(\operatorname{dtw}(G))$. In particular this allows the construction of several disjoint concentric directed cycles in $G$ by exploiting the structure of the grid, Eulerianness, and planarity. We follow their ideas and show that the entire construction can be done in polynomial time to yield $\Omega(\operatorname{dtw}(H) / \Delta(H))$ concentric disjoint cycles.

The final step is to find many disjoint paths that cross the concentric cycles from the inner cycle to the outer cycle and many disjoint paths from the outer cycle to the inner cycle. We show that we can find such paths via some ideas in [24] but with the additional property that these paths originate at the terminals. The collection of concentric cycles with these crossing paths is our desired crossbar and we also obtain the required property that the terminals are linked to this crossbar. We note that [24] have to do considerable work to obtain the cylindrical grid while we are satisfied with the constant congestion properties of the cycles plus paths (see Fig.1).

In the end, we arrive at the following statement whose proof is presented in Section 3.

- Theorem 3. Given a plane directed Eulerian graph $G$ of maximum in-degree at most $\Delta$ and an $\alpha$-node-well-linked set $X$ in $G$ with $|X|=\Omega\left(\Delta^{2} / \alpha\right)$, one can in polynomial time find a set of $\Omega(\alpha|X| / \Delta)$ concentric cycles going in the same direction (i.e., all clockwise or all counter-clockwise), sets $Y^{+}, Y^{-} \subseteq X$ of size $\left|Y^{+}\right|=\left|Y^{-}\right|=\Omega\left(\alpha^{2}|X| / \Delta^{2}\right)$ each, and families $\mathcal{P}^{+}$and $\mathcal{P}^{-}$of node-disjoint paths, such that either

1. none of the cycles enclose any vertex of $Y^{+} \cup Y^{-}$, the family $\mathcal{P}^{+}$consists of $\left|Y^{+}\right|$ node-disjoint paths from $Y^{+}$to the innermost cycle, and the family $\mathcal{P}^{-}$consists of $\left|Y^{-}\right|$ node-disjoint paths from the innermost cycle to $Y^{-}$; or 

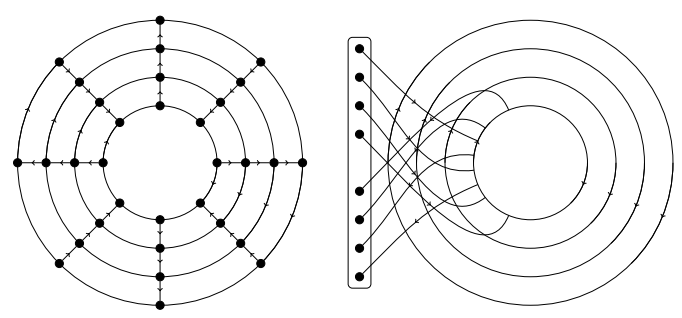

Figure 1 A cylinder (left) and a crossbar we obtain in our proof (right); the vertices in the rounded rectangle is a set of $\alpha$-well-linked terminals.

$$
\begin{array}{cr}
\max \sum_{i=1}^{k} x_{i} & \\
\sum_{p \in \mathcal{P}\left(s_{i}, t_{i}\right)} f(p)=x_{i} & i \in[k] \\
\sum_{p \in \mathcal{P}\left(t_{i}, s_{i}\right)} f(p)=x_{i} & i \in[k] \\
\sum_{p: v \in p} f(p) \leq 1 & v \in V(G) \\
f(p) \geq 0 & \\
x_{i} \in[0,1] & i \in \mathcal{P} \\
& i \in[k]
\end{array}
$$

Figure 2 Relaxation Sym-Dir-NDP LP.

2. all cycles enclose $Y^{+} \cup Y^{-}$, the family $\mathcal{P}^{+}$consists of $\left|Y^{+}\right|$node-disjoint paths from $Y^{+}$ to the outermost cycle, and the family $\mathcal{P}^{-}$consists of $\left|Y^{-}\right|$node-disjoint paths from the outermost cycle to $Y^{-}$.

Although we are inspired by [24], in the proof of Theorem 3 we use different methodology based on well-linked sets. We also point out that there are significant technical hurdles in working with directed graphs and treewidth. For instance, one can prove that if an undirected graph has treewidth $k$ then it has $\Omega(k / \log k)$ disjoint cycles. This is closely related to the well-known Erdos-Posa theorem [20]. Relating treewidth and disjoint cycles in directed graphs is significantly harder and was resolved in [35] (and also via the more recent result [28]) but the quantitative relationship is weak and far from the known lower bounds.

Using Theorem 3, we show the following statement, which in turn, together with Theorem 2, immediately yields Theorem 1 .

- Theorem 4. There is an $\mathcal{O}\left(\Delta^{2} / \alpha^{3}\right)$ approximation with congestion 5 for Sym-Dir-NDP in instances for which the input digraph is planar and Eulerian with maximum degree $\Delta$, and the terminals are $\alpha$-node-well-linked for some $\alpha \leq 1$.

In this extended abstract, we focus on proving Theorem 3 on constructing the crossbar in Section 3, and we defer the remaining details and proofs to a longer version of this paper.

\section{Preliminaries on LP Relaxation and plane Eulerian digraphs}

LP relaxation. Our algorithm uses a standard multicommodity flow relaxation for the problem given in Figure 2. We use $\mathcal{P}(u, v)$ to denote the set of all paths in $G$ from $u$ to $v$, for each ordered pair $(u, v)$ of nodes. Our assumption that the pairs $\mathcal{M}$ form a matching ensures that the sets $\mathcal{P}\left(s_{i}, t_{i}\right), \mathcal{P}\left(t_{i}, s_{i}\right), \mathcal{P}\left(s_{j}, t_{j}\right)$ and $\mathcal{P}\left(t_{j}, s_{j}\right)$ are pairwise disjoint. Let $\mathcal{P}=\bigcup_{i=1}^{k}\left(\mathcal{P}\left(s_{i}, t_{i}\right) \cup \mathcal{P}\left(t_{i}, s_{i}\right)\right)$. The LP has a variable $f(p)$ for each path $p \in \mathcal{P}$ representing the amount of flow on $p$. For each (unordered) pair $s_{i} t_{i} \in \mathcal{M}$, the LP has a variable $x_{i}$ denoting the total amount of flow routed for the pair (in the corresponding IP, $x_{i}$ denotes whether the pair is routed or not). The LP imposes the symmetry constraint that there is a flow from $s_{i}$ to $t_{i}$ of value $x_{i}$ and a flow from $t_{i}$ to $s_{i}$ of value $x_{i}$. Additionally, the LP has 
capacity constraints that ensure that the total amount of flow on paths using a given node is at most one. ${ }^{2}$

It is convenient to assume that the pairs $\mathcal{M}$ form a matching on $\mathcal{T}$ and each terminal is a leaf of $G$, i.e., it is attached to a single neighbor using an edge in each direction. As shown in [7], these properties can be ensured as follows. Given an instance $(G, \mathcal{M})$ with terminal $\mathcal{T}$, we create a new instance $\left(G^{\prime}, \mathcal{M}^{\prime}\right)$ by attaching a new leaf neighbor $t^{\prime}$ to every $t \in \mathcal{T}$ with $\operatorname{arcs}\left(t, t^{\prime}\right)$ and $\left(t^{\prime}, t\right)$, and move the terminal $t$ to $t^{\prime}$. Given a solution to the LP relaxation on $(G, \mathcal{M})$, we can easily find a solution of at least half of the value by extending the flow along $\operatorname{arcs}\left(t, t^{\prime}\right)$ and $\left(t^{\prime}, t\right)$; the loss of the flow is due to potential capacity violation at vertex $t$ that is now counted twice along the flow paths. If we obtain an integral solution in $\left(G^{\prime}, \mathcal{M}^{\prime}\right)$ (i.e., a routing of some pairs from $\mathcal{M}^{\prime}$ ) with congestion $c>1$, by shortening the paths we obtain a routing with the same congestion in $(G, \mathcal{M})$.

Plane Eulerian Digraphs: First, let us recall the following lemma that encapsulates the main property of Eulerian digraphs that make them similar to undirected graphs.

- Lemma 5. Let $G$ be an Eulerian digraph of maximum in-degree $\Delta$, let $A, B \subseteq V(G)$, and let $\ell$ be an integer. If there exist $(\Delta+1) \ell+1$ undirected vertex-disjoint paths from $A$ to $B$ in $G$, then there exist $\ell+1$ directed ones as well.

We also need some notation with respect to planar embeddings. Let $\Pi$ denote the euclidean plane. For a closed Jordan curve $\gamma$ and a point $p \in \Pi \backslash \gamma$, by $\zeta_{p}(\gamma) \in \mathbb{Z}$ we denote the element of the fundamental group of $\Pi \backslash\{p\}$ where $\gamma$ belongs (with the convention that a clockwise cycle around $p$ is the +1 element). A Jordan curve $\gamma$ is in general position with respect to the plane graph $G$ if it has finite number of intersections with $G$, its starting point and ending point do not belong to $G$, and whenever a point $p$ lies both on $\gamma$ and in the interior of an edge $e \in E(G)$, then $\gamma$ traverses the edge $e$ at this point. A face-edge curve in a plane digraph $G$ is a Jordan curve in general position that does not traverse any vertex of $G$.

For a curve $\gamma$ in general position with respect to $G$, we introduce the following notions. Assume $\gamma$ intersects an edge $e$ while going from a face $f$ to a face $f^{\prime}$. If $e$ has the face $f$ on the right and the face $f^{\prime}$ on the left, then we say that e crosses $\gamma$ from left to right and, otherwise, if $e$ has the face $f$ on the left and the face $f^{\prime}$ on the right, then we say that $e$ crosses $\gamma$ from right to left. By $\operatorname{cross}^{L \rightarrow R}(\gamma)$ and $\operatorname{cross}^{R \rightarrow L}(\gamma)$ we denote the number of times an edge crosses $\gamma$ from left to right and from right to left, respectively; note that in these numbers we may count one edge multiple times, one for each moment $\gamma$ crosses the edge.

For a vertex $v$ in a digraph $G$, an imbalance of $v$ is the number $\operatorname{imb}_{G}(v):=\operatorname{indeg}_{G}(v)-$ $\operatorname{outdeg}_{G}(v)$. A graph is balanced if $\operatorname{imb}_{G}(v)=0$ for every $v \in V(G)$, and Eulerian if it is additionally weakly connected. Furthermore, let the imbalance of a curve $\gamma$ in a general position with respect to $G$ be $\operatorname{imb}(\gamma)=\operatorname{cross}^{L \rightarrow R}(\gamma)-\operatorname{cross}^{R \rightarrow L}(\gamma)$. A standard argument shows the following:

\footnotetext{
2 There is a subtle issue here with regards to the capacity usage at the endpoints of a path. In the integral solution, a pair of paths, one from $s_{i}$ to $t_{i}$ and one from $t_{i}$ to $s_{i}$, is regarded as using the vertex $s_{i}$ only once and using the vertex $t_{i}$ only once; in other words, such a pair can be seen as a simple cycle passing through $s_{i}$ and $t_{i}$. To simulate it in the LP relaxation, we consider that the starting vertex belongs to a flow path, but the ending vertex does not belong to it. Alternatively, we can assume that a flow path uses only half of the capacities at its endpoints; these interpretations are equivalent due to the symmetry of the demands.
} 

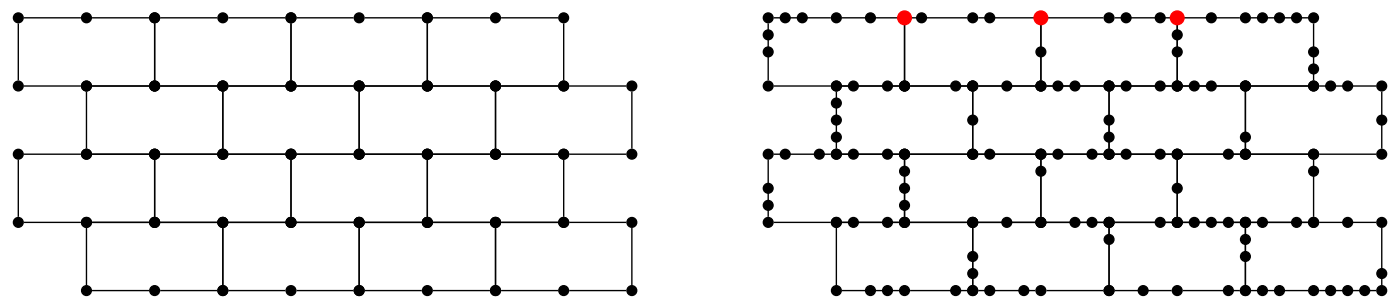

Figure $3 \mathrm{~A}$ wall (left) and a subdivided wall (right). The red vertices denote the interface of the wall.

- Lemma 6. Let $\gamma$ be a closed face-edge curve in a plane digraph $G$. Then

$$
\operatorname{imb}(\gamma)=\sum_{v \in V(G)} \zeta_{v}(\gamma) \cdot \operatorname{imb}_{G}(v)
$$

We also need the following flow/cut duality.

- Lemma 7. Given a plane digraph $G$, two distinguished faces $f^{\text {in }}$ and $f^{\text {out }}$, and an integer $k$, one can in linear time find either:

1. a family of directed vertex-disjoint cycles $C_{1}, C_{2}, \ldots, C_{k}$, all having $f^{\text {in }}$ to the right and $f^{\text {out }}$ to the left;

2. a curve $\gamma$ in general position with respect to $G$, that starts in $f^{\text {in }}$, ends in $f^{\text {out }}$, intersects at most $k$ vertices, and satisfies $\operatorname{cross}^{L \rightarrow R}(\gamma)=0$.

\section{The crossbar construction for Eulerian graphs}

We first remark that the two outcomes of Theorem 3 are the same if one considers embeddings on the sphere, while on the plane they differ only by the choice of the outer face of the embedding. Furthermore, note that all paths in $\mathcal{P}^{+}$and in $\mathcal{P}^{-}$intersect every constructed concentric cycle due to planarity.

In the proof of Theorem 3, without loss of generality we assume that every vertex of $X$ is incident to one outgoing arc and one incoming arc, and these two arcs have the same second endpoint: We can achieve it by creating a pendant vertex $x^{\prime}$ for every $x \in X$, connected to $x$ with $\operatorname{arcs}\left(x, x^{\prime}\right)$ and $\left(x^{\prime}, x\right)$; note that the well-linkedness of $X$ may drop to $\alpha /(\alpha+1)$ in this manner. Consequently, every cycle and path has no vertices in $X$ (except for possibly some endpoints); henceforth we will implicitly use this property multiple times.

Obtaining an undirected grid. We start by applying the construction for undirected planar graphs from [8]. Let $G^{\mathrm{UN}}$ be the underlying undirected graph of $G$. Clearly, $X$ is $\alpha$-nodewell-linked in $G^{\mathrm{UN}}$ and thus $G^{\mathrm{UN}}$ has (undirected) treewidth $\Omega(\alpha|X|)$. Hence we can obtain a large grid minor linked to the terminals $X$ using the following theorem of [8]. In what follows, it is notationally more convenient to work with subdivided walls as subgraphs, instead of minors. A $t \times t$ wall and a subdivided wall are shown in Figure 3. The $(t-1)$ vertices of degree three in the top row of a $t \times t$ wall $\Gamma$ are called the interface of the wall, denoted $I_{\Gamma}$.

- Theorem 8 (Theorem 4.5 of [8]). For every constant $\alpha \leq 1$, given an undirected planar graph $H$ and an $\alpha$-node-well-linked set $X$ in $H$, one can in polynomial time find an integer $t=\Omega(\alpha|X|)$, a subdivided $t \times t$ wall $\Gamma$ in $H$, and a family of $t$ node-disjoint paths connecting $X$ and the interface of $\Gamma$. 


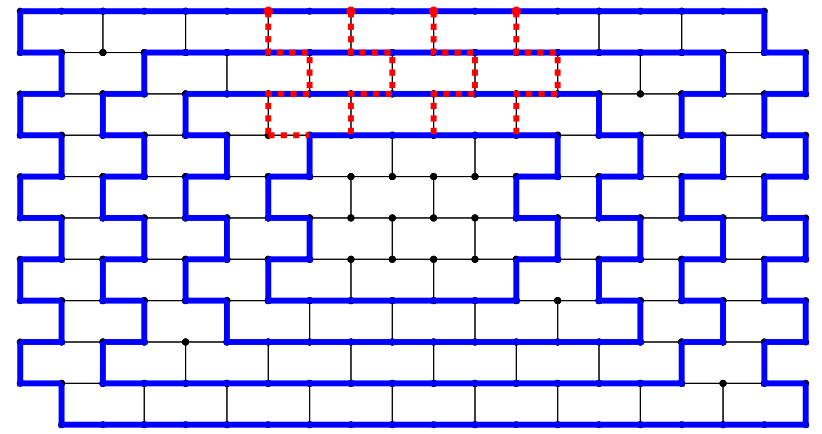

Figure 4 Illustration of Corollary 9. The cycles are blue, while the paths are dashed red.

In our construction we do not need the entire structure of a subdivided wall, but only part of it, as in the following immediate corollary (see Fig. 4).

- Corollary 9. One can in polynomial time find an integer $r=\Omega(\alpha|X|)$ and a sequence of node-disjoint concentric undirected cycles $C_{1}, C_{2}, \ldots, C_{r}$ in $G^{\mathrm{UN}}$, with $C_{1}$ being the outermost and $C_{r}$ being the innermost cycle, with the additional property that for every $1 \leq i \leq r$ there exists $r$ vertex-disjoint paths in $G^{\mathrm{UN}}$ from $X$ to $V\left(C_{i}\right)$.

Isles $\boldsymbol{S}^{\text {out }}$ and $\boldsymbol{S}^{\text {in }}$. Let us fix a choice of $r$ and cycles $C_{1}, C_{2}, \ldots, C_{r}$ stemming from Corollary 9. For a while, we work only with the undirected graph $G^{\mathrm{UN}}$. Our goal is to strengthen the requirement of the existence of many undirected paths between $X$ and the innermost and outermost cycles by getting more properties about their endpoints, so that we can use an argument similar to the one of [24] to reason about the existence of directed concentric cycles with similar connectivity towards $X$.

To this end, we identify two small connected parts of $G^{\mathrm{UN}}, S^{\text {out }}$ and $S^{\text {in }}$, one around $C_{1}$ and one around $C_{r}$. The parts will be large enough so that there is a substantial number of vertex-disjoint directed paths between them and $X$, but small enough so that they are placed very locally in the graph, and their boundary is small. This last property ensures that after deletion of these parts, the graph is close to Eulerian, and we can make use of Lemma 6 .

For a vertex set $Q \subseteq V\left(G^{\mathrm{UN}}\right)$, a vertex $v \notin Q$, and an integer $\ell \geq 2 \Delta$, we say that a vertex set $S$ is a $(v, Q, \ell)$-isle if $v \in S, G^{\mathrm{UN}}[S]$ is connected, $S \cap Q=\emptyset$, and $\left|N_{G^{\mathrm{UN}}}(S)\right| \leq \ell{ }^{3}$ We will rely on the following greedy procedure, that is inspired by the enumeration algorithm for important separators in parameterized complexity (cf. [12] and [18, Chapter 8]).

Lemma 10. Given a set $Q \subseteq V\left(G^{\mathrm{UN}}\right)$, a vertex $v \notin Q$, and an integer $\ell \geq 2 \Delta$, one can in $O\left(\ell^{3} n\right)$ time find an inclusion-wise maximal $(v, Q, \ell)$-isle.

Proof. We perform the following iterative procedure. Start with $S=\{v\}$; clearly, $S$ is a $(v, Q, \ell)$-isle, as $v \notin Q$ by assumption and the maximum in-degree of $G$ is $\Delta$. In an iterative step, we assume that $S$ is a $(v, Q, \ell)$-isle, and our goal is to check if $S$ is an inclusion-wise maximal one, or produce a $(v, Q, \ell)$-isle $S^{\prime}$ with $S \subsetneq S^{\prime}$.

\footnotetext{
3 We use the following notation with respect to neighborhoods. Let $G$ be an undirected graph, $x \in V(G)$, and $S \subseteq V(G)$. Then $N_{G}(x)$ is the set of neighbors of $x$ in $G, N_{G}[x]=\{x\} \cup N_{G}(x), N_{G}[S]=$ $\bigcup_{x \in S} N_{G}[x], N_{G}(S)=N_{G}[S] \backslash S, N_{G}^{2}[S]=N_{G}\left[N_{G}[S]\right]$, and $N_{G}^{2}(S)=N_{G}\left(N_{G}[S]\right)$.
} 
To this end, consider every $w \in N_{G^{\mathrm{UN}}}(S) \backslash Q$; note that, by the connectivity of $S^{\prime}$ and $S$, there exists such $w \in S^{\prime} \backslash S$ for every isle $S^{\prime}$ we are looking for. Collapse in $G^{\mathrm{UN}}$ the set $S \cup\{w\}$ into a single vertex $s$ and add a super-source vertex $t$ adjacent to all vertices of $Q$. Let $G^{\prime}$ be the resultingg (undirected) graph. Find a minimum $s-t$ vertex cut $Z$ in $G^{\prime}$, or conclude that such a minimum cut is of size larger than $\ell$; this can be done using $\mathcal{O}(\ell)$ rounds of the Ford-Fulkerson algorithm, taking total time $\mathcal{O}(\ell n)$. Moreover, within this time we can find the minimum cut closest to $t$, that is, the unique one with inclusion-wise maximal set of vertices remaining in the connected component with the vertex $s$ (cf. [18]).

If such a cut is found, let $S^{\prime}$ be the subset of vertices of $G$ corresponding to the connected component of $G^{\prime} \backslash Z$ containing the vertex $s$. Clearly, $N_{G^{\mathrm{UN}}}\left(S^{\prime}\right)=Z$, and $S^{\prime}$ is a $(v, Q, \ell)$-isle containing $S$ and $w$. Otherwise, we conclude that no $(v, Q, \ell)$-isle containing both $S$ and $w$ exists, since for every such isle $S^{\prime}$, the set $N_{G^{\mathrm{UN}}}\left(S^{\prime}\right)$ is an $s-t$ cut in $G^{\prime}$ of size at most $\ell$.

The computation for fixed $S$ and $w$ takes $\mathcal{O}(\ell n)$ time. Since $S$ is an $(v, Q, \ell)$-isle, there are at most $\ell$ vertices $w$ to try. Due to the fact that we always take the $s-t$ cut closest to $t$, the size of the set $N_{G^{\mathrm{UN}}}(S)$ strictly grows at every iteration (possibly except the first one, when $S=\{v\}$ ). Consequently, they are at most $\ell+1$ iterations of the procedure, and the running time bound follows.

We pick an arbitrary vertex $v^{\text {out }}$ on $C_{1}$ and an arbitrary vertex $v^{\text {in }}$ on $C_{r}$, and use Lemma 10 for both these vertices, the set $Q:=X$, and threshold $\ell:=\lfloor r /(4 \Delta+2)\rfloor$; recall that $|X|=\Omega\left(\Delta^{2} / \alpha\right)$ by the assumptions of Theorem 3 thus we may assume $\ell \geq 2 \Delta$. Let $S^{\text {out }}$ and $S^{\text {in }}$ be the two isles obtained. Since $\ell<r$, and every cycle $C_{i}$ is connected with $r$ vertex-disjoint paths to $X$, no cycle $C_{i}$ is contained in either $S^{\text {out }}$ or $S^{\text {in }}$. Since an isle is connected, we obtain the following.

- Lemma 11. The isle $S^{\text {out }}$ does not contain any vertex that is enclosed by $C_{\ell+1}$, and the isle $S^{\text {in }}$ does not contain any vertex that is not strictly enclosed by $C_{r-\ell}$.

Proof. The proofs for $S^{\text {in }}$ and $S^{\text {out }}$ are symmetrical, so we just focus here on the case of $S^{\text {out }}$. Assume to the contrary that $S^{\text {out }}$ contains a vertex enclosed by $C_{\ell+1}$. Since $v^{\text {out }} \in S^{\text {out }}$ and by the connectity of $S^{\text {out }}, S^{\text {out }}$ contains a vertex from every cycle $C_{i}, 1 \leq i \leq \ell+1$. Since $\left|N_{G^{\mathrm{UN}}}\left(S^{\text {out }}\right)\right| \leq \ell$, for some $1 \leq i \leq \ell+1$ we have that $V\left(C_{i}\right)$ is completely contained in $S^{\text {out }}$. However, recall that there are $r>\ell$ vertex-disjoint paths in $G^{\mathrm{UN}}$ connecting $C_{i}$ with $X$. This contradicts the facts that $S^{\text {out }} \cap X=\emptyset$ and $\left|N_{G^{\mathrm{UN}}}\left(S^{\text {out }}\right)\right| \leq \ell$.

By Lemma 11, the isles $S^{\text {out }}$ and $S^{\text {in }}$ are somewhat local in the graph: they do not go too deep into the set of cycles $C_{1}, C_{2}, \ldots, C_{r}$. On the other hand, recall that they are inclusion-wise maximal isles; by the next lemma, this ensures that they are connected by a large number of vertex-disjoint undirected paths to the set $X$. Let $W^{\text {out }}=N_{G^{\mathrm{UN}}}^{2}\left[S^{\text {out }}\right]$ and $W^{\text {in }}=N_{G \text { UN }}^{2}\left[S^{\text {in }}\right]$.

- Lemma 12. In $G^{\mathrm{UN}}$, there are $\ell+1$ node-disjoint undirected paths connecting $W^{\text {out }}$ and $X$ and $\ell+1$ node-disjoint undirected paths connecting $W^{\text {in }}$ and $X$.

Proof. By symmetry, we can focus on the case of $W^{\text {out }}$. The intuition is as follows: if there does not exist a sufficient amount of desired node-disjoint paths, then the corresponding cut would allow us to construct a strictly larger isle, a contradiction to the maximality of $S^{\text {out }}$. In some sense, $N_{G^{\mathrm{UN}}}\left(S^{\text {out }}\right)$ is the "last bottleneck" of size at most $\ell$ between $v^{\text {out }}$ and $X$, and, after passing it, we should have more than $\ell$ paths between $X$ and $N_{G^{\mathrm{UN}}}^{2}\left[S^{\text {out }}\right]=W^{\text {out }}$.

Formally, assume the contrary of the lemma statement; by Menger's theorem, there exist vertex sets $A, B \subseteq V\left(G^{\mathrm{UN}}\right)$ such that $A \cup B=V\left(G^{\mathrm{UN}}\right),|A \cap B| \leq \ell, W^{\text {out }} \subseteq A, X \subseteq B$, and no edge of $G^{\mathrm{UN}}$ has one endpoint in $A \backslash B$ and the second endpoint in $B \backslash A$. 
Recall that $S^{\text {out }} \cap X=\emptyset$ by the definition of an isle, while $N_{G^{\mathrm{UN}}}^{2}\left[S^{\text {out }}\right]=W^{\text {out }} \subseteq A$. Hence we may assume that $\left(N_{G^{\mathrm{UN}}}\left[S^{\text {out }}\right] \backslash X\right) \subseteq(A \backslash B)$, as removing all vertices of $N_{G^{\mathrm{uN}}}\left[S^{\text {out }}\right] \backslash X$ from $B$ would not invalidate any of the properties of the pair $(A, B)$. Recall also that $G^{\mathrm{UN}}\left[S^{\text {out }}\right]$ is connected; let $S_{A}$ be the vertex set of the connected component of $G^{\mathrm{UN}} \backslash(A \cap B)$ containing $S^{\text {out }}$. Clearly, $S_{A} \subseteq A \backslash B$, so $S_{A} \cap X=\emptyset$. Furthermore, $N_{G}$ un $\left(S_{A}\right) \subseteq A \cap B$, so $\left|N_{G^{\text {UN }}}\left(S_{A}\right)\right| \leq \ell$. As $S^{\text {out }} \subseteq S_{A}$, by the maximality of $S^{\text {out }}$, we infer that $S_{A}=S^{\text {out }}$. Since $N_{G^{\mathrm{UN}}}\left[S^{\text {out }}\right] \backslash X \subseteq S_{A}$, we infer that $N_{G^{\mathrm{UN}}}\left(S^{\text {out }}\right) \subseteq X$. However, this is a contradiction, as $G^{\mathrm{UN}}$ is connected and $S^{\text {out }} \subsetneq V\left(G^{\mathrm{UN}}\right) \backslash X$.

Finding directed concentric cycles. We now use the undirected cycles $C_{1}, C_{2}, \ldots, C_{r}$ to find a large number of node-disjoint directed concentric cycles separating $S^{\text {in }}$ and $S^{\text {out }}$. Recall that $\ell:=\lfloor r /(4 \Delta+2)\rfloor$.

- Lemma 13. One can in polynomial time find $\lceil\ell / 2\rceil$ node-disjoint directed concentric cycles, all going in the same direction (all clockwise or all counter-clockwise), such that all vertices of $S^{\text {in }}$ are strictly enclosed by the innermost cycle, and all vertices of $S^{\text {out }}$ are not enclosed by the outermost cycle, or vice versa, with the roles of $S^{\text {in }}$ and $S^{\text {out }}$ swapped.

Proof. Denote $G^{\prime}=G \backslash\left(N_{G^{\mathrm{UN}}}\left[S^{\text {out }}\right] \cup N_{G^{\mathrm{UN}}}\left[S^{\text {in }}\right]\right)$. Let $f^{\text {out }}$ and $f^{\text {in }}$ be the faces of $G^{\prime}$ that contain $S^{\text {out }}$ and $S^{\text {in }}$, respectively; by Lemma 11 , the cycle $C_{\lceil r / 2\rceil}$ remains in $G^{\prime}$ and $f^{\text {out }} \neq f^{\text {in }}$. Furthermore, the vertices of $N_{G^{\mathrm{UN}}}^{2}\left(S^{\text {out }}\right)$ lie on the face $f^{\text {out }}$ of $G^{\prime}$, and the vertices of $N_{G^{\text {UN }}}^{2}\left(S^{\text {in }}\right)$ lie on the face $f^{\text {in }}$. We apply Lemma 7 twice to the graph $G^{\prime}$ and the requirement of $\ell$ cycles, once for the pair of faces $\left(f^{\text {out }}, f^{\text {in }}\right)$ and once for the pair $\left(f^{\text {in }}, f^{\text {out }}\right)$. If at least one of the applications returns a family of cycles, then we are done, as every cycle encloses either $S^{\text {in }}$ or $S^{\text {out }}$. Thus, we are left with the case when both the applications return a curve; let us denote these curves $\gamma_{1}$ and $\gamma_{2}$, respectively.

Before we proceed to the formal calculations leading to a contradiction, let us give some intuition. The curves $\gamma_{1}$ and $\gamma_{2}$ are very skewed in terms of the directions of edges crossing it: only edges in one direction are allowed, while in the second direction only $\ell$ vertices are allowed, and every vertex is of maximum in-degree $\Delta$. The locality of isles $S^{\text {out }}$ and $S^{\text {in }}$ (Lemma 11) implies that $\gamma_{1}$ and $\gamma_{2}$ cross most of the cycles $C_{i}$; consequently, they need to cross much more than $\ell \Delta$ arcs in one direction. However, the graph $G^{\prime}$ is very close to an Eulerian one, as we have a bound of $\ell$ on the size of the boundary of $S^{\text {out }}$ and $S^{\text {in }}$. This leads to a contradiction with Lemma 6 for a closed curve being essentially a concatenation of $\gamma_{1}$ and $\gamma_{2}$.

Formally, let us first modify the curve $\gamma_{1}$ to obtain a face-edge curve $\gamma_{1}^{\prime}$ as follows: whenever $\gamma_{1}$ crosses a vertex $v$, we move it slightly to avoid $v$, at the cost of intersecting some of the arcs incident to $v$. Since the maximum in-degree and out-degree of $G$ (and thus $\left.G^{\prime}\right)$ is at most $\Delta$, we have that $\operatorname{cross}^{L \rightarrow R}\left(\gamma_{1}^{\prime}\right) \leq \Delta(\ell-1)$. Similarly, we obtain a curve $\gamma_{2}^{\prime}$ with $\operatorname{cross}^{L \rightarrow R}\left(\gamma_{2}^{\prime}\right) \leq \Delta(\ell-1)$. Since $\gamma_{1}^{\prime}$ starts in $f^{\text {out }}$ and ends in $f^{\text {in }}$, while $\gamma_{2}^{\prime}$ starts in $f^{\text {in }}$ and ends in $f^{\text {out }}$, we can concatenate these curves (without introducing any new intersection with $G^{\prime}$ ) and obtain a closed face-edge curve $\gamma^{\prime}$. This curve $\gamma^{\prime}$ visits both $f^{\text {out }}$ and $f^{\text {in }}$, and satisfies

$$
\operatorname{cross}^{L \rightarrow R}\left(\gamma^{\prime}\right) \leq 2 \Delta(\ell-1)
$$

By Lemma 11, the undirected cycles $C_{\ell+2}, C_{\ell+3}, \ldots, C_{r-\ell-1}$ remain in $G^{\prime}$, and both $\gamma_{1}^{\prime}$ and $\gamma_{2}^{\prime}$ need to cross at least one edge of each of these cycles. Consequently,

$$
\operatorname{cross}^{L \rightarrow R}\left(\gamma^{\prime}\right)+\operatorname{cross}^{R \rightarrow L}\left(\gamma^{\prime}\right) \geq 2(r-2 \ell-2) .
$$


By merging (1) and (2), and by the choice of $\ell$, we obtain that:

$$
-\operatorname{imb}\left(\gamma^{\prime}\right)=\operatorname{cross}^{R \rightarrow L}\left(\gamma^{\prime}\right)-\operatorname{cross}^{L \rightarrow R}\left(\gamma^{\prime}\right) \geq 2(r-2 \ell-2-2 \Delta(\ell-1))>4 \Delta \ell .
$$

On the other hand, note that every vertex of $G^{\prime}$ with a non-zero imbalance is a former neighbor of a vertex of $N_{G^{\mathrm{UN}}}\left(S^{\text {out }} \cup S^{\text {in }}\right)$. As there are at most $2 \ell$ vertices in $N_{G^{\mathrm{UN}}}\left(S^{\text {out }} \cup S^{\text {in }}\right)$, and every such vertex has in-degree and out-degree bounded by $\Delta$, we have

$$
\sum_{v \in V\left(G^{\prime}\right)}\left|\operatorname{imb}_{G^{\prime}}(v)\right| \leq \sum_{u \in N_{G^{\mathrm{UN}}}\left(S^{\text {out }} \cup S^{\text {in }}\right)} \operatorname{indeg}_{G}(u)+\operatorname{outdeg}_{G}(u) \leq 4 \Delta \ell .
$$

Equations (3) and (4) stand in contradiction with Lemma 6.

Finishing the crossbar construction. Let $C_{1}^{\prime}, \ldots, C_{\lceil\ell / 2\rceil}^{\prime}$ be the concentric directed cycles found by Lemma 13; by symmetry, assume they all enclose $S^{\text {in }}$. Let $q:=\lceil\ell / 4\rceil$. We consider two cases, depending on whether at least half of the vertices of $X$ are enclosed by $C_{q}^{\prime}$ or not. The two cases correspond to the two symmetric outcomes of Theorem 3. In what follows, we describe only the first case, when at least half of the vertices of $X$ are enclosed by $C_{q}^{\prime}$, and we use cycles $C_{1}^{\prime}, C_{2}^{\prime}, \ldots, C_{q}^{\prime}$ and vertex-disjoint paths from $X$ to $W^{\text {out}}$; the second case is completely symmetric, but uses cycles $C_{q+1}^{\prime}, C_{q+2}^{\prime}, \ldots, C_{\ell}^{\prime}$ and paths from $X$ to $W^{\text {in }}$.

Consider the set of $\ell+1$ paths in $G^{\mathrm{UN}}$ connecting $W^{\text {out }}$ and $X$, whose existence is promised by Lemma 12, and let $X^{\text {out }}$ be the set of the endpoints of the paths. The vertices in $X^{\text {out }}$ may not be enclosed by $C_{q}^{\prime}$. Our goal is to find a different set of vertices that are enclosed by $C_{q}^{\prime}$ such that they have disjoint paths to $W^{\text {out }}$; we use well-linkedness of $X$ for this purpose. As $\ell+1 \leq|X| / 2$ and the set $X$ is $\alpha$ node-well-linked, for every set $X^{\prime} \subseteq X$ of $\ell+1$ vertices enclosed by $C_{q}^{\prime}$, there exist $\alpha(\ell+1)$ node-disjoint paths connecting $X^{\text {out }}$ and $X^{\prime}$. By combining these paths with the paths connecting $W^{\text {out }}$ and $X^{\text {out }}$, we obtain a flow that sends $\alpha(\ell+1) / 2$ amount of flow in $G^{\mathrm{UN}}$ with unit node capacities from $X^{\prime}$ to $W^{\text {out }}$, with $1 / 2$ originating in every vertex in $X^{\prime}$. Consequently, there exists a set $Y \subseteq X$ of size at least $\alpha(\ell+1) / 2$, whose vertices are all enclosed by $C_{q}^{\prime}$, and such that there exist $|Y|$ node-disjoint paths in $G^{\mathrm{UN}}$ connecting $Y$ and $W^{\text {out }}$.

By Lemma 5, there exist at least $(\alpha \ell-2) /(2(\Delta+1))$ node-disjoint directed paths from $Y$ to $W^{\text {out }}$ (we let $Y^{+} \subset Y$ denote the end points of these paths) and the same amount of node-disjoint directed paths from $W^{\text {out }}$ to $Y$ (we let $Y^{-} \subset Y$ denote the end points of these paths). Recall that $\ell=\Theta(\alpha|X| / \Delta)$, thus $(\alpha \ell-2) /(2(\Delta+1))=\Theta\left(\alpha^{2}|X| / \Delta^{2}\right)$. As no vertex of $W^{\text {out }}$ is strictly enclosed by $C_{1}^{\prime}$, these paths, together with the cycles $C_{1}^{\prime}, C_{2}^{\prime}, \ldots, C_{q}^{\prime}$, form the desired structure. This concludes the proof of Theorem 3 .

\section{$4 \quad$ Concluding Remarks}

Our main technical contribution in this paper is to show that a planar directed graph has a constant congestion routing structure of size $\Omega(h / \operatorname{polylog}(h))$, where $h=\operatorname{dtw}(G)$. This structural result was motivated by the algorithmic problem of routing symmetric demands in directed graphs. Recent results, in the undirected graph setting, have demonstrated effectively the inherent synergy between approximation algorithms for routing problems and structural results in graph theory related to treewidth. The work in [7] and here are steps towards extending this synergy to directed graphs. The directed graph setting is significantly more challenging, however, and progress in this direction could yield several new benefits. We raise some open problems below. 
- Does a planar directed graph with treewidth $h$ have a constant congestion crossbar of size $\Omega(h)$. This would strengthen our result. In particular, is there a cylindrical grid minor of size $\Omega(h)$ ?

- The techniques in this paper could likely be extended to directed graphs that can be embedded on a bounded genus surface, and more generally to directed graphs whose undirected support graph is from a proper minor-closed family. The ideas of well-linked decomposition and degree-reduction do not rely on planarity. Moreover, there is a linear relationship between treewidth and the size of a grid-minor in undirected graphs from a proper minor-closed family [19].

- Does a general directed graph with treewidth $h$ have a constant congestion crossbar of size $\Omega(h / \operatorname{polylog}(h))$ ? Is there a cylindrical grid minor of size $\Omega\left(h^{\delta}\right)$ for some fixed $\delta>0$ ?

\section{References}

1 M. Andrews. Approximation algorithms for the edge-disjoint paths problem via Raecke decompositions. In Proc. of IEEE FOCS, pages 277-286, 2010.

2 M. Andrews, J. Chuzhoy, V. Guruswami, S. Khanna, K. Talwar, and L. Zhang. Inapproximability of edge-disjoint paths and low congestion routing on undirected graphs. Combinatorica, 30(5):485-520, 2010.

3 C. Chekuri and J. Chuzhoy. Large-treewidth graph decompositions and applications. In Proc. of ACM STOC, pages 291-300, 2013.

4 C. Chekuri and J. Chuzhoy. Polynomial bounds for the grid-minor theorem. In Proc. of ACM STOC, pages 60-69, 2014.

5 C. Chekuri and J. Chuzhoy. Degree-3 treewidth sparsifiers. In Proc. of ACM-SIAM SODA, pages 242-255, 2015.

6 C. Chekuri and A. Ene. Poly-logarithmic approximation for maximum node disjoint paths with constant congestion. In Proc. of ACM-SIAM SODA, pages 326-341, 2013.

7 C. Chekuri and A. Ene. The all-or-nothing flow problem in directed graphs with symmetric demand pairs. Mathematical Programming, pages 1-24, 2014.

8 C. Chekuri, S. Khanna, and F.B. Shepherd. Multicommodity flow, well-linked terminals, and routing problems. In Proc. of ACM STOC, pages 183-192, 2005.

9 C. Chekuri, S. Khanna, and F.B. Shepherd. An $O(\sqrt{n})$ approximation and integrality gap for disjoint paths and unsplittable flow. Theory of Computing, 2(7):137-146, 2006.

10 Chandra Chekuri, Sreeram Kannan, Adnan Raja, and Pramod Viswanath. Multicommodity flows and cuts in polymatroidal networks. SIAM Journal on Computing, 44(4):912-943, 2015. Preliminary version in Proc. of ITCS, 2012.

11 Chandra Chekuri, Sanjeev Khanna, and F Bruce Shepherd. The all-or-nothing multicommodity flow problem. SIAM Journal on Computing, 42(4):1467-1493, 2013. Preliminary version in Proc. of ACM STOC, 2004.

12 J. Chen, Y. Liu, S. Lu, B. O'Sullivan, and I. Razgon. A fixed-parameter algorithm for the directed feedback vertex set problem. Journal of the ACM, 55(5), 2008.

13 J. Chuzhoy. Routing in undirected graphs with constant congestion. In Proc. of ACM STOC, pages 855-874, 2012.

14 J. Chuzhoy. Excluded grid theorem: Improved and simplified. In Proc. of ACM STOC, pages $645-654,2015$.

15 J. Chuzhoy, V. Guruswami, S. Khanna, and K. Talwar. Hardness of routing with congestion in directed graphs. In Proc. of ACM STOC, pages 165-178, 2007.

16 J. Chuzhoy, D.H.K. Kim, and S. Li. Improved approximation for node-disjoint paths in planar graphs. In Proc. of ACM STOC, 2016. To appear. 
17 J. Chuzhoy and S. Li. A polylogarithimic approximation algorithm for edge-disjoint paths with congestion 2. In Proc. of IEEE FOCS, 2012.

18 M. Cygan, F. Fomin, L. Kowalik, D. Loksthanov, D. Marx, M. Pilipczuk, M. Pilipczuk, and S. Saurabh. Parameterized Algorithms. Springer, 2014. In print.

19 E. D. Demaine and M. Hajiaghayi. Linearity of grid minors in treewidth with applications through bidimensionality. Combinatorica, 28(1):19-36, 2008.

20 P. Erdos and L. Pósa. On independent circuits contained in a graph. Canadian Journal of Mathematics, 17:347-352, 1965.

21 G. Even, J. Naor, S. Rao, and B. Schieber. Divide-and-conquer approximation algorithms via spreading metrics. Journal of the ACM, 47(4):585-616, 2000.

22 Steven Fortune, John Hopcroft, and James Wyllie. The directed subgraph homeomorphism problem. Theoretical Computer Science, 10(2):111-121, 1980.

23 T. Johnson, N. Robertson, P. D. Seymour, and R. Thomas. Directed tree-width. Journal of Combinatorial Theory, Series B, 82(1):138-154, 2001.

24 T. Johnson, N. Robertson, P. D. Seymour, and R. Thomas. Excluding a grid minor in digraphs. Manuscript, http://arxiv.org/abs/1510.00473, 2001.

25 S. Kamath, S. Kannan, and P. Viswanath. Network capacity under traffic symmetry: Wireline and wireless networks. IEEE Transactions on Information Theory, 60(9):54575469, 2014.

26 S. Kannan and P. Viswanath. Capacity of multiple unicast in wireless networks: A polymatroidal approach. IEEE Transactions on Information Theory, 60(10):6303-6328, 2014.

27 K. Kawarabayashi and S. Kreutzer. An excluded grid theorem for digraphs with forbidden minors. In Proc. of ACM-SIAM SODA, pages 72-81, 2014.

28 K. Kawarabayashi and S. Kreutzer. The directed grid theorem. In Proc. of ACM STOC, 2015.

29 R. Khandekar, S. Rao, and U. Vazirani. Graph partitioning using single commodity flows. Journal of the ACM, 56(4):19, 2009.

30 P. N. Klein, S.A. Plotkin, S. Rao, and E. Tardos. Approximation algorithms for Steiner and directed multicuts. Journal of Algorithms, 22(2):241-269, 1997.

31 S. G. Kolliopoulos and C. Stein. Approximating disjoint-path problems using packing integer programs. Mathematical Programming, 99(1):63-87, 2004.

32 A. Louis. Cut-matching games on directed graphs. CoRR, abs/1010.1047, 2010.

33 S. Rao and S. Zhou. Edge disjoint paths in moderately connected graphs. SIAM Journal on Computing, 39(5):1856-1887, 2010.

34 B. Reed. Introducing directed tree width. Electronic Notes in Discrete Mathematics, 3:222229, 1999.

35 B. Reed, N. Robertson, P. D. Seymour, and R. Thomas. Packing directed circuits. Combinatorica, 16(4):535-554, 1996.

36 N. Robertson, P. D. Seymour, and R. Thomas. Quickly excluding a planar graph. Journal of Combinatorial Theory, Series B, 62(2):323-348, 1994.

37 P. D. Seymour. Packing directed circuits fractionally. Combinatorica, 15(2):281-288, 1995. 